\title{
Optimisation of piezoelectric cantilever energy harvesters including non-linear effects
}

\author{
R Patel, S McWilliam, A A Popov \\ Dept. of Mechanical, Materials and Manufacturing Engineering, University of \\ Nottingham, Nottingham, NG7 2RD, UK \\ E-mail: Rupesh.Patel@nottingham.ac.uk
}

\begin{abstract}
The paper proposes a versatile non-linear model for the prediction of piezoelectric energy harvester performance. The presented model includes (i) material non-linearity, for both substrate and piezoelectric layers, and (ii) geometric nonlinearity incorporated by assuming in-extensibility and accurately representing beam curvature. The addition of a sub-model, which utilises the transfer matrix method to predict eigenfrequencies and eigenvectors for segmented beams, allows for accurate optimisation of piezoelectric layer coverage. A validation of the overall theoretical model is performed through experimental testing on both uniform and non-uniform samples manufactured in-house.

For the harvester composition used in this work, the magnitude of material non-linearity exhibited by the piezoelectric layer is 35 times greater than that of the substrate layer. It is also observed that material non-linearity, responsible for reductions in resonant frequency with increases in base acceleration, is dominant over geometric non-linearity for standard piezoelectric harvesting devices. Lastly, over the tested range, energy loss due to damping is found to increase in a quasi-linear fashion with base acceleration.

During an optimisation study on piezoelectric layer coverage, results from the developed model were compared with those from a linear model. Unbiased comparisons between harvesters were realised by using devices with identical natural frequencies - created by adjusting device substrate thickness. Results from three studies, each with a different assumption in mechanical damping variations, are presented. Findings showed that, depending on damping variation, a non-linear model is essential for such optimisation studies with each model predicting vastly differing optimum configurations.
\end{abstract}

Energy harvesting, Piezoelectric beam, Geometric non-linearity, Material non-linearity, Coverage optimisation 


\section{Introduction}

Over the last decade there has been a growing increase in research in the field of vibrational energy harvesting. The principle is to convert ambient vibrational energy into electrical energy with practical devices primarily targeted at replacing or supplementing the batteries used to power wireless sensors for condition monitoring, e.g. a tyre pressure sensor [1]. The use of piezoelectric materials is seen to be the most effective transduction method [2]; a statement which is reflected by piezoelectric devices receiving the most interest. For general information on vibrational energy harvesting the reader is referred to compendium in the form of a recent book [3]. However, more specifically for piezoelectric energy harvesters the reader is directed to Piezoelectric Energy Harvesting [4].

There has been a great deal of work on the linear modelling of cantilever piezoelectric harvesters. Sodano et al. [5] used the Rayleigh-Ritz procedure for estimating the power output from a cantilever mounted piezoelectric generator. Ertuk and Inman [6] later developed a distributed-parameter electromechanical model for energy harvesters. Validation was also completed by the authors with theoretical results in good agreement with experimental data [7]. Patel et al. expanded on this by accurately incorporating the effects of non-uniform beams created by altering piezoelectric layer coverage. Utilisation of the model showed vast improvements in performance are achievable with experimental data indicating model validity [8]. However, despite the level of interest in the area, reliable non-linear modelling of piezoelectric harvesters validated through detailed experimental work is generally lacking.

Models exist to simulate energy harvesters operating in conditions or arrangements where non-linearity is induced intentionally. This approach is utilised to assist in overcoming a major limitation to vibrational energy harvester usability - a limited operational bandwidth [9]. In early research, Ramlan et al. [10] theoretically investigated the effects of introducing non-linearity via spring hardening with system behaviour represented in the form of the Duffing equation. System bandwidth was found to increase, with the peak magnitude of generated voltage remaining on a similar level to that predicted by a linear model. Similar theoretical and experimental research, whereby non-linearity is induced through the use of magnets, can be found in $[11,12]$ to name a few. More recent advances on the use of magnets are presented by Tang et al. in $[13,14]$. The authors propose the use of a magnetic oscillator in-place of a fixed magnet with experimental results indicating a $100 \%$ improvement in bandwidth and a $42 \%$ improvement in peak power at an acceleration level of $2 \mathrm{~ms}^{-2}$ over both fixed magnet and linear harvester designs [13]. The piezoelectric energy harvester-magnetic oscillator design is realised through the inclusion of an additional cantilever with a magnetic proof mass, above the conventional energy harvester cantilever. Readers are directed to [14] for detailed information, through experimental work, on the use of magnets in improving the functionality of energy harvesters through monostable/bistable device designs and 
also designs targeted at frequency-up-conversion for use in low frequency applications. An alternate method for intentionally inducing non-linearity is through the use of a static axial pre-load. Masana and Daqaq [15] accurately modelled and experimentally validated such a scenario for piezoelectric energy harvesters. Findings indicated that axial pre-load can be beneficial in the improvement of both device bandwidth, by introducing non-linearity, and peak power, by increasing the electrical damping.

Non-linearity naturally exists in vibrating systems undergoing large deflections (geometric non-linearity), and when certain materials are involved in harvester composition - particularly associated with the piezoelectric layer. As shown by Joshi in [16], non-linear constitutive equations can be used to define material nonlinear behaviour, with Crespo da Silva and Glynn [17] providing fundamental work on geometric non-linearity in beams. Few works can be found which attempt to incorporate these effects in relation to the modelling of energy harvesters, e.g. [18, 19, 20], with experimental validation providing confidence in the developed models. Stanton et al. provide ground work for the modelling of inherent piezoelectric material non-linearities in energy harvesters [19]. Model validation is also provided through experimental testing on an off-the-shelf bimorph device. The model is restricted to the analysis of uniform samples, with symmetry through the thickness, where harvesters are comprised of layers being identical in length. The effects of substrate material non-linearity and geometric non-linearity are not considered in [19]. The representation of such factors is seen to be important in the modelling of certain types of energy harvester, namely thin film flexible energy harvesters. Mutsuda et al. [21] have proposed an ocean power generator which comprises of underwater hanging flexible harvesters attached to an elastic floating unit. The floating unit can generate electric power from wave oscillations and wave breaking whilst hanging units utilise vibrations created by underwater currents, vortices and oscillation. Naturally, as a result of low oscillatory frequencies occurring in an ocean environment ( $\approx 0.8-1.2 \mathrm{~Hz}$ ), the harvester must be manufactured from flexible material. Silicone rubber for the substrate layer(s) and polyvinylidene flouride (PVDF) for the piezoelectric layer(s) are the materials of choice. The usage of such flexible devices is not only limited to the ocean environment and can be utilised in, for example, wind flutter applications - exacting energy on/near bridges. The materials used in the manufacture of such devices, and the large deflections which they undertake, indicate the requirement for an improved analytical non-linear model. Our study is heavily motivated by these devices. It is important to be able to predict how non-linear behaviour affects the frequency response of the harvester (the extent of resonance shift and peak magnitude reduction) in order to ensure the harvester is designed to operate most efficiently at the dominant excitation frequency.

In this paper a versatile non-linear model will be developed which has the ability to accurately predict piezoelectric energy harvester performance. The model will include a transfer matrix sub-model to determine the dynamic behaviour of segmented beams which are generated when altering the coverage of the piezoelectric layer. Coupling with energy expressions for segmented harvester structures will allow for the optimisation of 
devices in terms of their piezoelectric layer coverage. This was not considered before, e.g. in [18] or [19], but is seen to be essential when designing performance/cost efficient devices. Substrate material non-linearity will also be included in model development. This factor is highly important as the model is expected to be used on thin film flexible devices in the near future. Additionally, the curvature of the beam which is required to incorporate geometric non-linearity, will be obtained through differential geometry. The paper is laid out in the following manner. Section 2 will disclose information on the modelling approach and the embodiment of material and geometric non-linearities.

Hamilton Extended Principle along with the calculus of variations will be used to obtain the final two equations of the motion (transverse displacement and voltage). This will be followed by a substantial section on model validation through experimental work, Section 3. Results obtained from four different samples manufactured 'in-house', with various lengths of piezoelectric material, will be presented here. Section 4 illustrates the importance of using the non-linear model during device optimisation over a linear model in certain operating conditions. Finally, the paper will end with closing remarks and suggestions for future work.

\section{Modelling a piezoelectric harvester system including various non-linearities}

In this section details behind the theoretical modelling of energy harvesters are presented, including both geometric and material non-linearities. To increase model versatility by allowing for alternations to piezoelectric layer coverage, a component of the model will utilise the transfer matrix method [22]. The accurate prediction of natural frequencies and mode shapes will result from such an approach. Electrical aspects of the system will comprise solely of a load resistor with previous work on more realistic circuitry, for example, storing generated energy in a capacitor, provided previously in [8].

\subsection{Energy harvester}

The general methodology behind model development is similar to that undertaken by several other researchers, i.e. [1, 23]. Material non-linearity is introduced in the form of additional terms found in constitutive equations, and geometric non-linearity will result from an inextensible beam assumption. Extended Hamilton's principle, along with the calculus of variations, is used to obtain equations of motion in the time domain.

A schematic of the structure is provided in Figure 1. The considered harvester consists of a composite two-layer Euler-Bernoulli beam, with piezoelectric material perfectly bonded to a substrate layer. $x_{1}$ represents the distance of the piezoelectric layer from the clamped end, and $x_{2}$ is the length of the piezoelectric layer. The current Euler-Bernoulli beam assumption, in which shear deformation and rotary effects are neglected, is reasonable for the vast majority of expected harvester geometries, and 


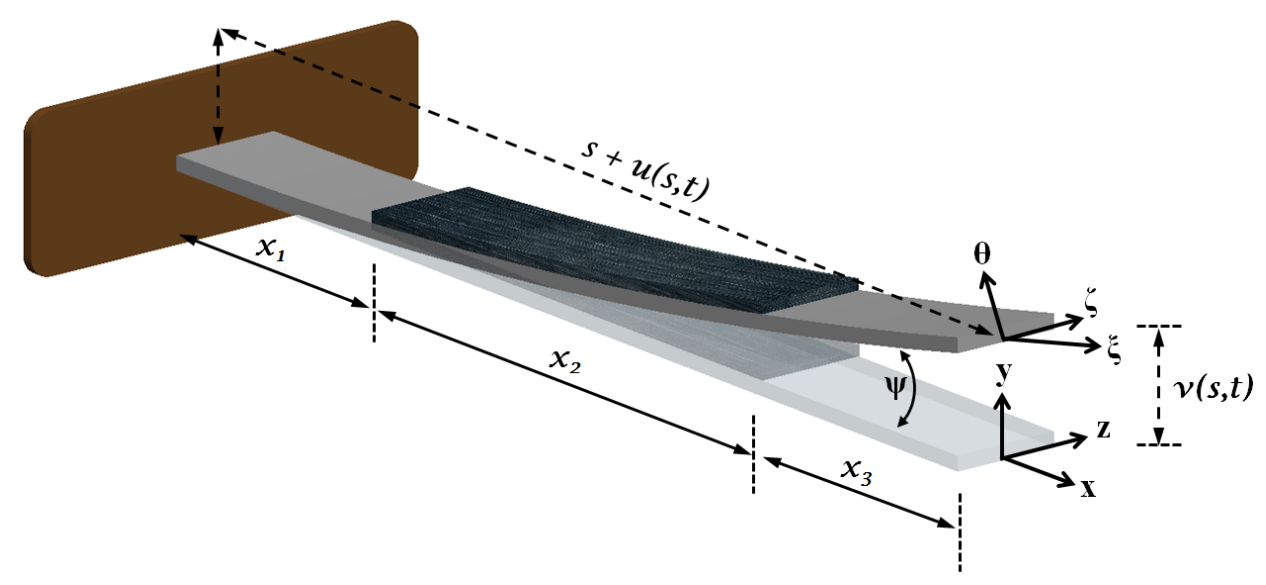

Figure 1: A schematic on the notation and displacements used during non-linear model development.

justified by Dietl et al. in [24]. Other notation in Figure 1 is taken in conjunction with [23], with the Newtonian inertial co-ordinate system represented by $(x, y, z)$ and the local co-ordinate system represented by $(\xi, \theta, \zeta)$.

2.1.1. Constitutive equations The constitutive equation of a material is used to relate axial stress, $\sigma_{11}$, to axial strain, $\varepsilon_{11}$, and, in addition for the piezoelectric material, the electric displacement, $D_{3}$, to axial strain. Herein, index 3 refers to the $y$-direction, i.e. through the thickness of the material, and index 1 refers to the $x$-direction, i.e. parallel to the beam length. For the piezoelectric material [23] one has

$$
\begin{aligned}
& \sigma_{11}^{p}=E_{p} \varepsilon_{11}^{p}+\frac{\mu_{1}}{2}\left(\varepsilon_{11}^{p}\right)^{2}-E_{p} d_{31} E_{\text {field }}-\mu_{2} \varepsilon_{11}^{p} E_{\text {field }}, \\
& D_{3}=E_{p} d_{31} \varepsilon_{11}^{p}+\frac{\mu_{2}}{2}\left(\varepsilon_{11}^{p}\right)^{2}+\varepsilon_{33} E_{\text {field }},
\end{aligned}
$$

where superscript $p$ refers to the piezoelectric layer, $E_{p}$ is the piezoelectric material Young's modulus, and $E_{\text {field }}$ the electric field strength. $d_{31}$ is a piezoelectric material constant, and $\varepsilon_{33}$ is the material permittivity. The difference between the above equations (Equations (1) and (2)), and linear relationships used in for example [7], is the inclusion of higher order terms with constants $\mu_{1}$ and $\mu_{2}$. These terms represent non-linearity, and are both specific and unique to each 'batch' of piezoelectric material.

For the substrate material one has

$$
\sigma_{11}^{s}=E_{s} \varepsilon_{11}^{s}+\frac{\mu_{s 1}}{2}\left(\varepsilon_{11}^{s}\right)^{2},
$$

where superscript $s$ refers to the substrate layer and $E_{s}$ is the substrate material Young's modulus. Note how a coefficient of material non-linearity, $\mu_{s 1}$, has also been included for the substrate layer. As a result of experimental setup uncertainties, the importance of this term is realised in Section 3.1. However, as alluded to in the Introduction, its inclusion is paramount for the modelling and performance predictions of certain harvester compositions, work which is not presented here. 
Equations governing system response will be determined through application of the Hamilton Extended Principle. This requires both the Lagrangian of the system and work done on the system, where the Lagrangian refers to the difference between kinetic energy, $T$, and potential energy, $U$. An expression for each of these terms is obtained in the following subsections.

2.1.2. Potential energy The potential energy present in the system can be expressed as follows. Note how different segments of the beam are considered individually due to variations in material composition.

$$
\begin{aligned}
U=\frac{1}{2} \int_{0}^{x_{1}} \iint_{A_{s}}( & \left.\sigma_{11}^{s} \varepsilon_{11}^{s}\right) \mathrm{d} A \mathrm{~d} s+\frac{1}{2} \int_{x_{1}}^{x_{1}+x_{2}} \iint_{A_{s}}\left(\sigma_{11}^{s} \varepsilon_{11}^{s}\right) \mathrm{d} A \mathrm{~d} s+\frac{1}{2} \int_{x_{1}}^{x_{1}+x_{2}} \iint_{A_{p}}\left(\sigma_{11}^{p} \varepsilon_{11}^{p}\right) \mathrm{d} A \mathrm{~d} s \\
& +\frac{1}{2} \int_{x_{1}+x_{2}}^{L} \iint_{A_{s}}\left(\sigma_{11}^{s} \varepsilon_{11}^{s}\right) \mathrm{d} A \mathrm{~d} s+\frac{1}{2} \int_{0}^{L} E A(s)\left(u^{\prime}(s, t)+\frac{1}{2}\left(v^{\prime}(s, t)\right)^{2}\right)^{2} \mathrm{~d} s \\
& -\frac{1}{2} \int_{x_{1}}^{x_{1}+x_{2}} \iint_{A_{p}} E_{\text {field }}(t) D_{3} \mathrm{~d} A \mathrm{~d} s
\end{aligned}
$$

where $L$ is the length of the substrate layer, or $x_{1}+x_{2}+x_{3}$, $\mathrm{d} s$ is the small element length, and $A_{s}$ and $A_{p}$ are cross-sectional areas for the substrate and piezoelectric material respectively. Terms $v(s, t)$ and $u(s, t)$ refer to the transverse and longitudinal deflection respectively, see Figure 1, while $\left(^{\prime}\right)$ donates the derivative with respect to arc length, $s$. Note how, herein, for the deflection terms, the independent variables $s$ and $t$ are excluded for ease in reading. The expression for $E A(s)$ must take into account nonuniform material distribution, realised through the use of Heaviside functions:

$$
\begin{aligned}
E A(s)= & (H(s-0)-H(s-L)) E_{s} b_{s} t_{s} \\
& +\left(H\left(s-x_{1}\right)-H\left(s-x_{1}-x_{2}\right)\right) E_{p} b_{p} t_{p} .
\end{aligned}
$$

Strain in the beam can be expressed in terms of the distance from the neutral axis, $y$, and beam curvature, $\rho$, viz.:

$$
\varepsilon_{11}=y \rho,
$$

where $\rho$ is obtained using differential geometry, see for example [25], and given by:

$$
\rho=v^{\prime \prime}+v^{\prime \prime} u^{\prime}-v^{\prime} u^{\prime \prime}
$$

with higher powers approximated as:

$$
\begin{aligned}
& \rho^{2} \approx\left(v^{\prime \prime}\right)^{2}+2\left(v^{\prime \prime}\right)^{2} u^{\prime}-2 v^{\prime \prime} v^{\prime} u^{\prime \prime}, \\
& \rho^{3} \approx\left(v^{\prime \prime}\right)^{3} .
\end{aligned}
$$

Utilising Equations (1)-(3) and Equations (6)-(8), in addition to the assumption that electric field is uniform throughout the piezoelectric thickness, $t_{p}$, i.e. $E_{\text {field }}(t)=$ 
$-V(t) / t_{p}$, transforms Equation (4) to:

$$
\begin{aligned}
U=\frac{1}{2} \int_{0}^{L} & \left\{\left(K_{1}(s)-K_{2}(s) V(t)\right)\left(\left(v^{\prime \prime}\right)^{2}+2\left(v^{\prime \prime}\right)^{2} u^{\prime}-2 v^{\prime \prime} v^{\prime} u^{\prime \prime}\right)\right. \\
& +K_{3}(s)\left(\left(v^{\prime \prime}\right)^{3}\right)-K_{4}(s)\left(v^{\prime \prime}+v^{\prime \prime} u^{\prime}-v^{\prime} u^{\prime \prime}\right) V(t) \\
& \left.+E A(s)\left(u^{\prime}+\frac{1}{2}\left(v^{\prime}\right)^{2}\right)^{2}-K_{5}(s) V^{2}(t)\right\} \mathrm{d} s
\end{aligned}
$$

where the terms $K_{1}$ through $K_{5}$ are given by:

$$
\begin{aligned}
K_{1}(s)= & \left(H(s-0)-H\left(s-x_{1}\right)\right) E_{s} I_{s}+\left(H\left(s-x_{1}\right)-H\left(s-x_{1}-x_{2}\right)\right) E_{s} I_{s 1} \\
& +\left(H\left(s-x_{1}\right)-H\left(s-x_{1}-x_{2}\right)\right) E_{p} I_{p 1} \\
& +\left(H\left(s-x_{1}-x_{2}\right)-H(s-L)\right) E_{s} I_{s}, \\
K_{2}(s)= & \frac{3}{2}\left(H\left(s-x_{1}\right)-H\left(s-x_{1}-x_{2}\right)\right)\left(\frac{\mu_{2} I_{p 1}}{t_{p}}\right), \\
K_{3}(s)= & \frac{\mu_{s 1}}{2}(H(s-0)-H(s-L)) I_{s 2}+\frac{\mu_{1}}{2}\left(H\left(s-x_{1}\right)-H\left(s-x_{1}-x_{2}\right)\right) I_{p 2}, \\
K_{4}(s)= & \left(H\left(s-x_{1}\right)-H\left(s-x_{1}-x_{2}\right)\right)\left(2 E_{p} d_{31} b_{p}\right)\left(t_{s}+\frac{t_{p}}{2}-\bar{y}\right), \\
K_{5}(s)= & \left(H\left(s-x_{1}\right)-H\left(s-x_{1}-x_{2}\right)\right)\left(\frac{b_{p} \varepsilon_{33}}{t_{p}}\right) .
\end{aligned}
$$

where $\bar{y}$ is the location of the neutral axis from the bottom of the substrate layer. $I_{s}$, $I_{s 1}, I_{p 1}$ and $I_{p 2}$ are provided by

$$
\begin{aligned}
& I_{s}=\frac{b_{s} t_{s}^{3}}{12} \\
& I_{s 1}=b_{s}\left[\left(t_{s}\right) \bar{y}^{2}-\left(t_{s}^{2}\right) \bar{y}+\frac{1}{3} t_{s}^{3}\right], \\
& I_{p 1}=b_{p}\left[\left(t_{p}\right) \bar{y}^{2}+\left(-2 t_{p} t_{s}-t_{p}^{2}\right) \bar{y}+\left(\frac{1}{3} t_{p}^{3}+t_{p}^{2} t_{s}+t_{p} t_{s}^{2}\right)\right], \\
& I_{s 2}=b_{s}\left[\frac{\left(t_{s}-\bar{y}\right)^{4}}{4}-\frac{(-\bar{y})^{4}}{4}\right] \\
& I_{p 2}=b_{p}\left[\frac{\left(t_{s}+t_{p}-\bar{y}\right)^{4}}{4}-\frac{\left(t_{s}-\bar{y}\right)^{4}}{4}\right] .
\end{aligned}
$$

where $t_{s}$ is the substrate thickness, and $b_{s}$ and $b_{p}$ refer to the substrate and piezoelectric layer width, respectively.

The in-extensibility condition is used to eliminate independent longitudinal vibrations, $u$, in Equation (10), by relating them to transverse vibrations, $v$. For the in-extensibility condition to be satisfied, the strain along the neutral axis [23], $\epsilon_{0}$, must equate to zero, where $\epsilon_{0}$ is given by:

$$
\varepsilon_{0}=\sqrt{\left(1+u^{\prime}\right)^{2}+\left(v^{\prime}\right)^{2}}-1 .
$$

Expansion, rearrangement and utilisation of Taylor's expansion leads to the following 
relationship:

$$
u^{\prime} \approx-\frac{1}{2}\left(v^{\prime}\right)^{2}
$$

Using the above relationship in Equation (10) results in a two-degree-of-freedom system, one dependant coordinate for displacement and the other for voltage. The next step is to obtain the system Lagrangian which requires an expression for the kinetic energy,

2.1.3. Kinetic energy and system Lagrangian The kinetic energy of such a system can be represented as:

$$
T=\frac{1}{2} \int_{0}^{L} m(s)\left[\left(-\int_{0}^{s} v^{\prime} \dot{v}^{\prime}\right)^{2}+\dot{v}^{2}\right] \mathrm{d} s .
$$

Note that the transverse-longitudinal relationship has been used to eliminate $\dot{u} . m(s)$ is the mass per unit length and can be defined as:

$$
m(s)=\rho_{s} A_{s}+\left(H\left(s-x_{1}\right)-H\left(s-x_{1}-x_{2}\right)\right) \rho_{p} A_{p},
$$

where in turn $\rho_{s}$ and $\rho_{p}$ are the substrate and piezoelectric material density respectively. The Lagrangian of the system can be defined as usual

$$
\mathcal{L}=T-U,
$$

which upon substitution of Equations (10) and (15) leads to:

$$
\begin{aligned}
\mathcal{L}=\frac{1}{2} \int_{0}^{L} & \left\{m(s)\left[\left(-\int_{0}^{s} v^{\prime} \dot{v}^{\prime}\right)^{2}+\dot{v}^{2}\right]\right. \\
& -\left(K_{1}(s)-K_{2}(s) V(t)\right)\left(\left(v^{\prime \prime}\right)^{2}+\left(v^{\prime \prime} v^{\prime}\right)^{2}\right)-K_{3}(s)\left(\left(v^{\prime \prime}\right)^{3}\right) \\
& \left.+K_{4}(s)\left(v^{\prime \prime}+\frac{1}{2} v^{\prime \prime}\left(v^{\prime}\right)^{2}\right) V(t)+K_{5}(s) V^{2}(t)\right\} \mathrm{d} s,
\end{aligned}
$$

In the following subsection, extended Hamilton's principle is utilised in-order to obtain the two equations of motion.

2.1.4. Governing equations of motion Before the calculus of variations can be used, an expression for external work done on the system, $\mathcal{W}$, requires formulation, and is a combination of base excitation, $\ddot{w}_{b}(t)$, and the electric potential energy:

$$
\mathcal{W}=\left(\iiint_{V_{s}} \rho_{s} v \mathrm{~d} V_{\text {total }}+\iiint_{V_{p}} \rho_{p} v \mathrm{~d} V_{\text {total }}\right) \ddot{w}_{b}(t)-V(t) q(t),
$$

where $q(t)$ is the electric charge generated by the energy harvester. Applying the extended Hamilton's principle, i.e.

$$
\int_{t_{1}}^{t_{2}} \delta(\mathcal{L}+\mathcal{W}) \mathrm{d} t=0
$$


and utilising the calculus of variations leads to the following equations of motion:

$$
\begin{aligned}
& m(s) \ddot{v}+\left[v^{\prime} \int_{L}^{s} m(s) \int_{0}^{s}\left(\ddot{v}^{\prime \prime} v^{\prime}+\left(\dot{v}^{\prime}\right)^{2}\right) \mathrm{d} s \mathrm{~d} s\right]^{\prime} \\
& +\left[\left(K_{1}(s)-K_{2}(s) V(t)\right) v^{\prime \prime}\right]^{\prime \prime}+\left[\left(\left(K_{1}(s)-K_{2}(s) V(t)\right) v^{\prime \prime} v^{\prime}\right)^{\prime} v^{\prime}\right]^{\prime} \\
& +\left[\frac{3}{2} K_{3}(s)\left(v^{\prime \prime}\right)^{2}\right]^{\prime \prime}-\left[\frac{1}{2} K_{4}(s) V(t)\right]^{\prime \prime}+\left[\frac{1}{2} K_{4}(s) v^{\prime \prime} v^{\prime} V(t)\right]^{\prime} \\
& -\left[\frac{1}{4} K_{4}(s)\left(v^{\prime}\right)^{2} V(t)\right]^{\prime \prime}=\left[\iiint_{V_{s}} \rho_{s} \mathrm{~d} V_{\text {total }}+\iiint \int_{V_{p}} \rho_{p} \mathrm{~d} V_{\text {total }}\right] \ddot{w}_{b}(t),
\end{aligned}
$$

and

$$
\begin{aligned}
\int_{0}^{L} & \left\{\frac{1}{2} K_{2}(s)\left(\left(v^{\prime \prime}\right)^{2}+\left(v^{\prime \prime} v^{\prime}\right)^{2}\right)+\frac{1}{2} K_{4}(s)\left(v^{\prime \prime}+\frac{1}{2} v^{\prime \prime}\left(v^{\prime}\right)^{2}\right)\right. \\
& \left.+K_{5}(s) V(t)\right\} \mathrm{d} s-q=0
\end{aligned}
$$

with the following associated boundary conditions:

$$
v(0, t)=v^{\prime}(0, t)=v^{\prime \prime}(L, t)=v^{\prime \prime \prime}(L, t)=0 .
$$

A classical modal analysis technique, namely Bubnov-Galerkin method, is used to obtain simplified ordinary differential equations from the existing partial differential equations previously shown. Using this approach the beam deflection, $v(s, t)$, is expressed as an infinite sum of products of normalised eigenvectors, $W_{r}(s)$, see Section 2.2 , and time dependant generalised co-ordinates, $\eta_{r}(t)$, viz.:

$$
v(s, t)=\sum_{r=1}^{\infty} W_{r}(s) \eta_{r}(t),
$$

where ' $r$ ' refers the mode number. Substituting this relationship into Equation (21) and (22) in addition to using orthonormality conditions, yields the following two governing equations of motions:

$$
\begin{aligned}
\ddot{\eta}_{r}(t) & +2 \gamma_{r} \omega_{r} \dot{\eta}_{r}(t)+\omega_{r}^{2} \eta_{r}(t)+C_{n 1}^{r} \eta_{r}^{2}(t)+C_{n 2}^{r} \eta_{r}^{3}(t)+C_{n 3}^{r} \ddot{\eta}_{r}(t) \eta_{r}^{2}(t) \\
& +C_{n 4}^{r} \dot{\eta}_{r}^{2}(t) \eta_{r}(t)-C_{n 5}^{r} V(t)-C_{n 6}^{r} \eta_{r}(t) V(t) \\
& +C_{n 7}^{r} \eta_{r}^{2}(t) V(t)-C_{n 8}^{r} \eta_{r}^{3}(t) V(t)=C_{n 9}^{r} \ddot{w}_{b}(t)
\end{aligned}
$$

and

$$
\begin{gathered}
C_{n 10}^{r} \eta_{r}(t) \dot{\eta}_{r}(t)+C_{n 11}^{r} \eta_{r}^{3}(t) \dot{\eta}_{r}(t)+C_{n 12}^{r} \dot{\eta}_{r}(t)+C_{n 13}^{r} \eta_{r}^{2}(t) \dot{\eta}_{r}(t) \\
+C_{n 14}^{r} \dot{V}(t)+\frac{V(t)}{R_{\text {load }}}=0
\end{gathered}
$$

Note how proportional damping, $\gamma_{r}$, has been introduced in Equation (25), to accommodate for energy dissipation from the system. $\omega_{r}$ refers to the natural frequency of the $r^{\text {th }}$ mode and is obtained using the transfer matrix method, see Section 2.2. In obtaining Equation (26), the rate of change in charge, $\dot{q}(t)$, i.e. current, has been expressed as $-V(t) / R_{\text {load }}$. This formulation is acceptable as these works will simulate 
and present results from energy harvesters connected directly to a load resistance. $C_{n 1}^{r}$ to $C_{n 14}^{r}$ are resulting constants independent of time, provided in Appendix 1.

Equations (25) and (26) represent the behaviour of unimorph vibrational piezoelectric energy harvesters under non-linearity inducing conditions. They can be solved simultaneously to determine transverse vibrations along the structure and voltage generated by the energy harvester. Numerical solving of the equations is achieved using ode solvers in MATLAB ${ }^{\circledR}$, through the SimULINK ${ }^{\circledR}$ interface [26], with data recorded once steady-state response has been achieved.

\subsection{Transfer matrix model for segmented structures}

The model presented in Section 2.1 requires knowledge of the natural frequencies and mode shapes of the harvester. In this section the transfer matrix method [22] is used to obtain this information for a segmented cantilever beam, by taking into account the length and position of the piezoelectric layer. As shown in Figure 2 the beam is split into three sections. The substrate material alone makes up sections 1 and 3, while section 2 comprises both piezoelectric and substrate materials. The notation used to define the mechanical forces and deformations at the modes of each element is shown in Figure 2 and 3 .

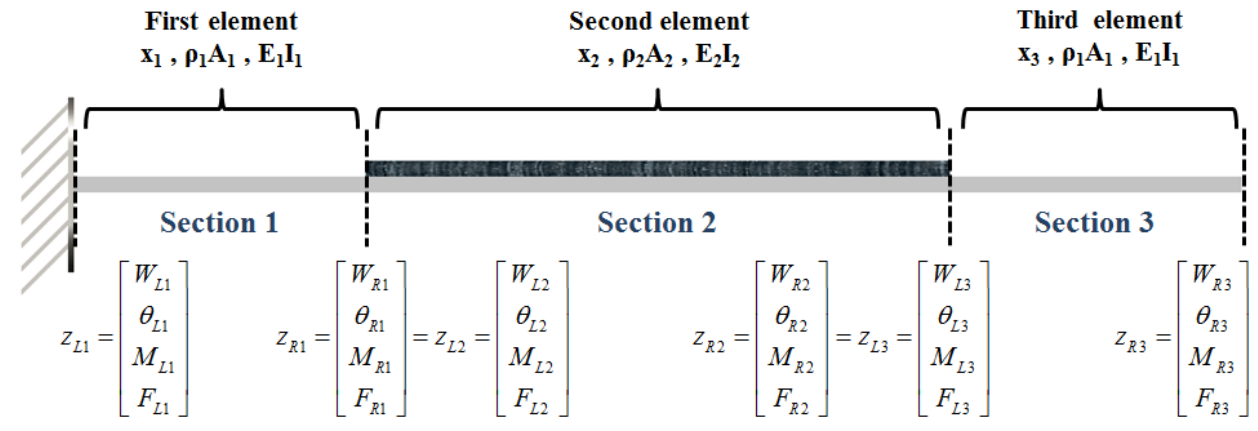

Figure 2: Element properties for beam sections. Each nodal joint has an associated displacement $(W)$, rotation $(\theta)$, moment $(M)$ and shear force $(F)$.

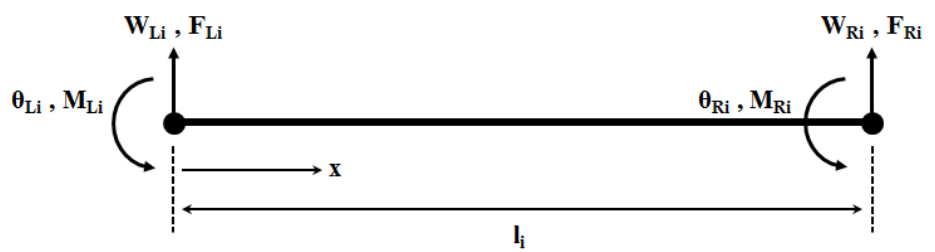

Figure 3: Notation adopted for information at nodal points of the $i^{\text {th }}$ beam element.

The exact beam function for the transverse motion of the $i^{\text {th }}$ section of a segmented beam is given by:

$W_{i}(x)=a \sin \left(\frac{\beta_{i} x}{l_{i}}\right)+b \cos \left(\frac{\beta_{i} x}{l_{i}}\right)+c \sinh \left(\frac{\beta_{i} x}{l_{i}}\right)+d \cosh \left(\frac{\beta_{i} x}{l_{i}}\right)$, 
where $a, b, c$ and $d$ are constants which are ordinarily determined using boundary conditions, $x$ is the distance from the left side of the beam segment and $l_{i}$ is the beam segment length. $\beta_{i}$ can be defined by:

$$
\beta_{i}=\omega^{0.5} l_{i}\left(\frac{\rho_{i} A_{i}}{E_{i} I_{i}}\right)^{0.25},
$$

where $E_{i}$ is the element Young's modulus, $I_{i}$ is the element area moment of inertia, $\rho_{i}$ is the element mass density and $A_{i}$ is the element cross-sectional area. The middle section is a composite piezoelectric/substrate beam and the properties of this element can be calculated using the equivalent flexural rigidity, viz.:

$$
(E I)_{\text {composite }}=E_{s} I_{y s}+E_{p} I_{y p},
$$

where the parallel axis theorem can be used to obtain $I_{y s}$ and $I_{y p}$, such that:

$$
\begin{aligned}
& I_{y s}=\frac{b_{s} t_{s}^{3}}{12}+b_{s} t_{s}\left(\bar{y}-\frac{t_{s}}{2}\right)^{2}, \\
& I_{y p}=\frac{b_{p} t_{p}^{3}}{12}+b_{p} t_{p}\left(\frac{t_{p}}{2}+t_{s}-\bar{y}\right)^{2} .
\end{aligned}
$$

The mechanical forces and deformations at the right-hand side of any beam element, $z_{R i}$, can be related to the left-hand side, $z_{L i}$, through the use of a transfer matrix, $\mathbf{U}_{\mathbf{i}}\left(l_{i}\right)$ [22], where,

$$
\left[\begin{array}{c}
W_{R i} \\
\theta_{R i} \\
M_{R i} \\
F_{R i}
\end{array}\right]=\mathbf{U}_{\mathbf{i}}\left(l_{i}\right)\left[\begin{array}{c}
W_{L i} \\
\theta_{L i} \\
M_{L i} \\
F_{L i}
\end{array}\right],
$$

and $\mathbf{U}_{\mathbf{i}}\left(l_{i}\right)$ is defined by:

$$
\mathbf{U}_{\mathbf{i}}\left(l_{i}\right)=\left[\begin{array}{cccc}
C_{0} & -l_{i} C_{1} & -\frac{l_{i}^{2} C_{2}}{E_{i} I_{i}} & -\frac{l_{i}^{3} C_{3}}{E_{i} I_{i}} \\
-\frac{\beta_{i}{ }^{4} C_{3}}{l_{i}} & C_{0} & \frac{l_{i} C_{1}}{E_{i} I_{i}} & \frac{l_{i}^{2} C_{2}}{E_{i} I_{i}} \\
-\frac{E_{i} I_{i} \beta_{i}{ }^{4} C_{2}}{l_{i}^{2}} & \frac{E_{i} I_{i} \beta_{i}{ }^{4} C_{3}}{l_{i}} & C_{0} & l_{i} C_{1} \\
-\frac{E_{i} I_{i} \beta_{i}{ }^{4} C_{1}}{l_{i}^{3}} & \frac{E_{i} I_{i} \beta_{i}{ }^{4} C_{2}}{l_{i}^{2}} & \frac{\beta_{i}{ }^{4} C_{3}}{l_{i}} & C_{0}
\end{array}\right] .
$$

Constants $C_{0}$ to $C_{3}$ are defined by:

$$
\begin{aligned}
& C_{0}=\frac{\cosh \left(\beta_{i}\right)+\cos \left(\beta_{i}\right)}{2}, \\
& C_{1}=\frac{\sinh \left(\beta_{i}\right)+\sin \left(\beta_{i}\right)}{2 \beta_{i}}, \\
& C_{2}=\frac{\cosh \left(\beta_{i}\right)-\cos \left(\beta_{i}\right)}{2 \beta_{i}{ }^{2}},
\end{aligned}
$$




$$
C_{3}=\frac{\sinh \left(\beta_{i}\right)-\sin \left(\beta_{i}\right)}{2 \beta_{i}{ }^{3}} .
$$

Full details on how the transfer matrix and related constants are derived is provided in [22]. For the general case, where piezoelectric material (of length shorter than the substrate layer) is centrally located on the beam, the overall transfer matrix of the system, $\mathbf{U}_{\text {overall }}$, is obtained from:

$$
\mathbf{U}_{\text {overall }}=\mathbf{U}_{\mathbf{3}}\left(x_{3}\right) \mathbf{U}_{\mathbf{2}}\left(x_{2}\right) \mathbf{U}_{\mathbf{1}}\left(x_{1}\right),
$$

where $\mathbf{U}_{1}, \mathbf{U}_{2}$ and $\mathbf{U}_{3}$ are the transfer matrices for each section. In some configurations certain sections are not present, i.e. for a conventional energy harvester the piezoelectric layer covers the full length of the beam and sections 1 and 3 are not present. In such cases, the corresponding transfer matrices can be removed from Equation (35). Since $\mathbf{U}_{\text {overall }}$ relates the clamped end of the structure to the free end, known boundary conditions (in a free vibration situation), $w_{\text {clamped }}=\theta_{\text {clamped }}=M_{\text {free }}=F_{\text {free }}=0$ can be used to obtain a matrix those determinant yields the natural frequencies of the system. The $2 \times 2$ matrix of interest is extracted from the bottom right corner of the $\mathbf{U}_{\text {overall }}$ matrix. Values of $\omega$ which produce determinant values of zero provide the natural frequencies of the system. Since mode shapes can be arbitrarily scaled, once natural frequencies are known the corresponding mode shapes are readily obtained by assuming one of the variables, i.e. clamped end shear force $F$, is unity. Following this the mode shapes are scaled as required, i.e. to the mass of the structure, allowing for the validity of orthonormality conditions used in Section 2.1.4.

\section{Validation through experimental testing}

In this section validation of the theoretical model presented in Section 2 is provided through the experimental testing of uniform and non-uniform samples. The energy harvesters, manufactured 'in-house' and of unimorph type, are comprised of an aluminium substrate layer and a lead zirconate titanate (PZT) piezoelectric layer. Adhesion is realised through a combination of DP460 epoxy and a small amount of silver conductive epoxy, with complete details behind the consistent manufacturing procedure found in [27]. In terms of the testing procedure, samples are mounted in a clamp attached to a Data Physics GW-V4 electromagnetic shaker providing base excitation. A Stanford Research Systems SR785 dynamic signal analyser is used to output a harmonic signal to the shaker via a standard amplifier. In addition to this, the analyser has the capability to record two unique signal inputs. One channel was always used to monitor base acceleration, in order to keep it fixed during frequency sweeping, while the second channel provided measurement for either voltage across a resistor or tip velocity. The base acceleration was measured using a PCB Piezotronic accelerometer, model number - 352C23, and the tip velocity measured with a PolyTec OFV-055 laser vibrometer, with velocity readings readily converted to displacement.

The coefficients of material non-linearity, i.e. $\mu_{1}$ and $\mu_{2}$, are unique to each 'batch' of piezoelectric material, and are not provided on the data sheets of material 
manufacturers. Such coefficients, along with $\mu_{s 1}$, are estimated using curve fitting techniques, as is standard practice in the field. Note, since all testing is performed close to fundamental frequencies, during theoretical simulation, the inclusion of solely the first vibrational mode, in Equations (25) and (26), is necessary, i.e. $r=1$. The fixed dimensions, i.e. layer widths and thickness, along with relevant material properties are provided in Table 1. Each sample in this section will be subjected to four increasing levels of base excitation $-0.5 \mathrm{~ms}^{-2}, 2.5 \mathrm{~ms}^{-2}, 5 \mathrm{~ms}^{-2}$ and $7.5 \mathrm{~ms}^{-2}$. The validation of the model is provided by estimating the coefficients from one sample alone and using the obtained magnitudes in the theoretical model to predict and compare results for other samples. Sample 1 (and a sample of only $\mathrm{Al}$ ) was used to obtained 1,2 and s1. The obtained magnitudes were then used to predict the behaviour of non-uniform samples 2 and 3 . The predicated behaviour will be shown to be in good agreement with experimental trends, indicting the model is valid for a range of piezoelectric material coverage.

Table 1: Structural dimensions and material properties of harvesters used throughout the paper.

\begin{tabular}{ll}
\hline \hline Parameter & Magnitude \\
\hline Al width $(\mathrm{mm})$ & 7 \\
$\mathrm{Al}$ thickness $(\mathrm{mm})$ & 0.87 \\
PZT width $(\mathrm{mm})$ & 7 \\
PZT thickness (mm) & 0.5 \\
Young's modulus of Al (GPa) & 69 \\
Density of Al (kg.m $\left.{ }^{-3}\right)$ & 2700 \\
Young's modulus of PZT (GPa) & 62.1 \\
Density of PZT (kg.m $\left.{ }^{-3}\right)$ & 7800 \\
\hline Piezoelectric constant, $d_{31}\left(\mathrm{~m} . \mathrm{V}^{-1}\right)$ & $-180 \times 10^{-12}$ \\
Permittivity, $\varepsilon_{33}^{S}\left(\mathrm{~F} . \mathrm{m}^{-1}\right)$ & $1.549 \times 10^{-8}$ \\
\hline \hline
\end{tabular}

\subsection{Solely a substrate layer}

Firstly the testing of only a substrate layer in the experiential setup was undertaken, i.e. a device comprising of no piezoelectric material. The results from this test are provided in Figure 4, superimposed with data from theoretical simulations. The sample used had an extended length of $43 \pm 1 \mathrm{~mm}$. Where extended length refers to the overhang length from the clamp face.

Experimental data in Figure 4 indicates a shift in the resonance from $295.9 \mathrm{~Hz}$ to $294.1 \mathrm{~Hz}$ when subjecting the sample to a base acceleration of $0.5 \mathrm{~ms}^{-2}$ and $7.5 \mathrm{~ms}^{-2}$ respectively. The magnitude of this shift can be accommodated for by setting $\mu_{s 1}$ to 


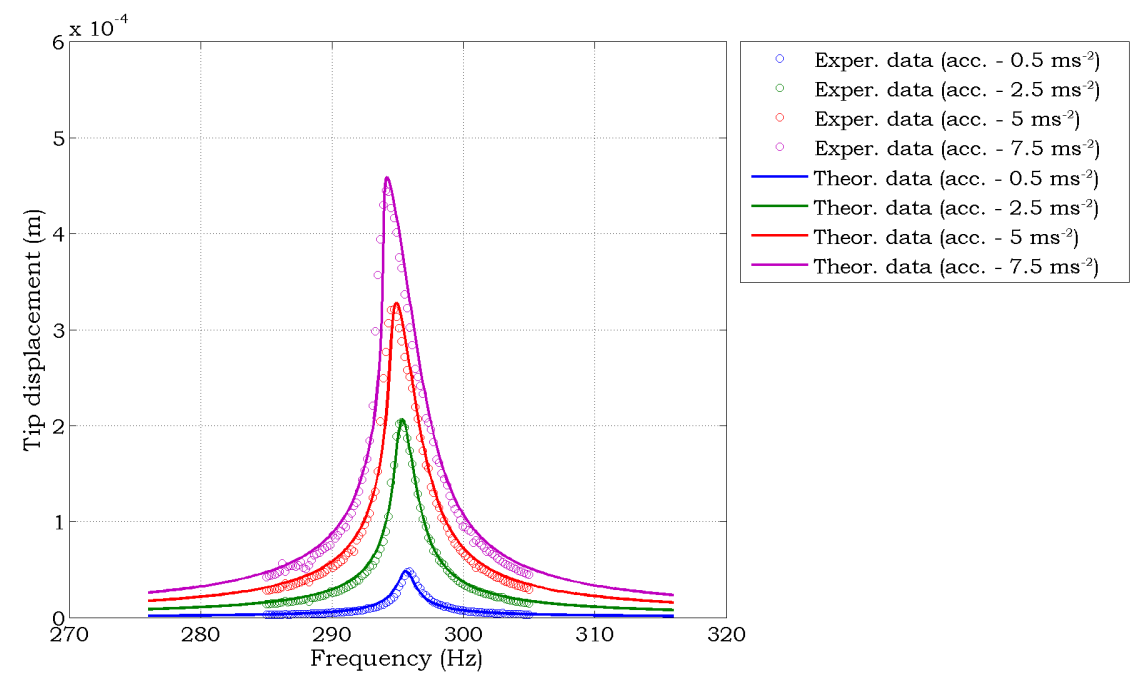

Figure 4: Experimental frequency responses of an aluminium beam at varying base acceleration levels with the inclusion of substrate material non-linearity.

$-2 \times 10^{13} \mathrm{~Pa}$; found through curve fitting in MATLAB ${ }^{\circledR}[26]$. The importance of including an additional form of non-linearity in conjunction to geometric and piezoelectric material non-linearity is evident from the example presented here. The softening phenomena would not have been reproducible had substrate material non-linearity been excluded from theoretical model formulation. The observed non-linearity could have resulted from other sources, such as boundary condition non-linearity or inertial non-linearity (see [28] for a detailed review on non-linearities in vibrating systems). However, the inclusion of substrate material non-linearity, albeit in a crude manner, will be shown in Section 3.2 to provide excellent experimental-theoretical agreement across a range of harvester samples.

As has been observed by previous researchers [29], the magnitude of mechanical damping experienced by the structure is dependant on the magnitude of acceleration, and found to be approximately linear across the tested range. Note that this is not in agreement with the non-linear damping assumption made by Stanton et al. in [19]. Damping magnitudes corresponding to theoretical plots in Figure 4 were extracted from experimental data using the half-power points method [31] and are shown in Figure 5.

In depth model development incorporating the variation in mechanical damping are beyond the scope of these works. However, briefly, for the experimental setup utilised, one can include, air flow damping, stick-slip at the clamped end, and material damping, to be the most likely causes of observed variations. Further details on variation in mechanical damping can be found in [30].

\subsection{Uniform and non-uniform energy harvesters in closed circuit conditions}

Following on, in this section the outcome of testing three energy harvester samples is presented, each comprising of differing piezoelectric layer lengths. Substrate and 


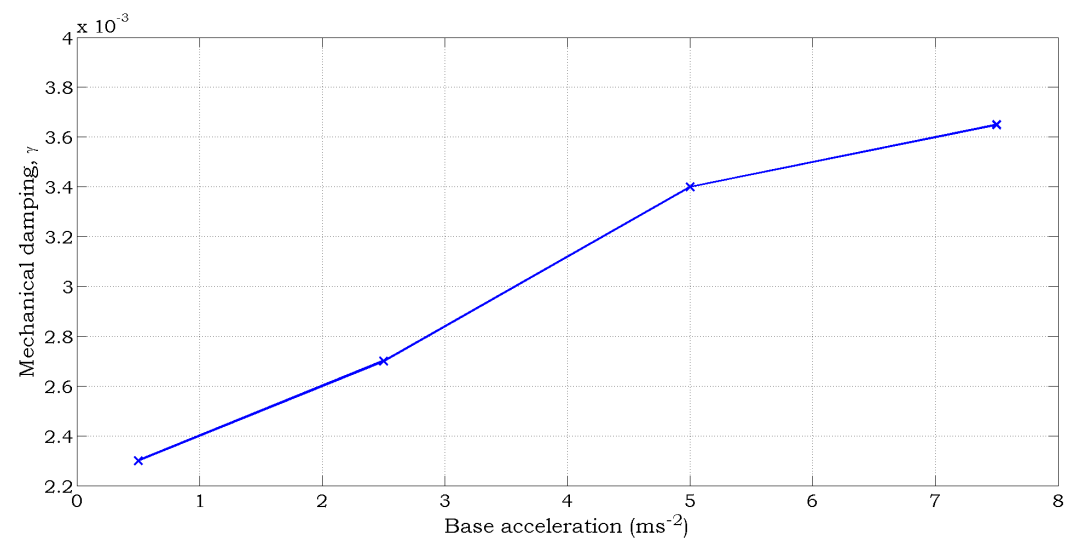

Figure 5: Dependency of mechanical damping on base acceleration for aluminium beam.

piezoelectric layer lengths for each sample are provided in Table 2. Note that for the non-uniform samples, the piezoelectric material length is reduced from the free end, i.e. clamping occurs on both piezoelectric and substrate layers, or $x_{1}=0$ in the theoretical model.

Table 2: Substrate and piezoelectric layer extended length for samples tested in this section.

\begin{tabular}{lll}
\hline \hline Sample & Al extended length $(\mathrm{mm})$ & PZT extended length $(\mathrm{mm})$ \\
\hline Uniform & $43.82 \pm 1$ & $43.82 \pm 1$ \\
Non-uniform 1 & $45.95 \pm 1$ & $18.15 \pm 1$ \\
Non-uniform 2 & $45.49 \pm 1$ & $9.77 \pm 1$ \\
\hline \hline
\end{tabular}

Results in this section are from three different samples tested in closed-circuit electrical conditions, i.e. $\mathrm{R}_{\text {load }}=0$. This eliminates one of the piezoelectric coefficients of non-linearity, namely $\mu_{2}$, facilitating the curve-fitting process. Note that the magnitude of the substrate material coefficient of non-linearity, $\mu_{s 1}$ previously found in Section 3.1, is used herein when generating theoretical results. Experimental data for the uniform conventional sample, superimposed with theoretical results, is provided in Figure 6.

Figure 6 shows that substantial non-linearity does exist in real situations and a softening phenomenon is witnessed once again. This observation will be a continual theme indicating that material non-linearity dominates over geometric non-linearity for this type of cantilever piezoelectric energy harvester. Numerically, the maximum deflection equates to 0.0017 the overhang length which reinforces reasoning behind why geometric non-linearity is negligible for this particular device. The resonant frequency of the structure was found to be $360.4 \mathrm{~Hz}$ at $0.5 \mathrm{~ms}^{-2}$, shifting to $355.3 \mathrm{~Hz}$ at $7.5 \mathrm{~ms}^{-2}$. Curve-fitting of data resulted in the coefficient $\mu_{1}$ requiring a magnitude of $-7 \times 10^{14} \mathrm{~Pa}$ (35 times than that for the substrate material) in order to replicate this $4.9 \mathrm{~Hz}$ shift. The 


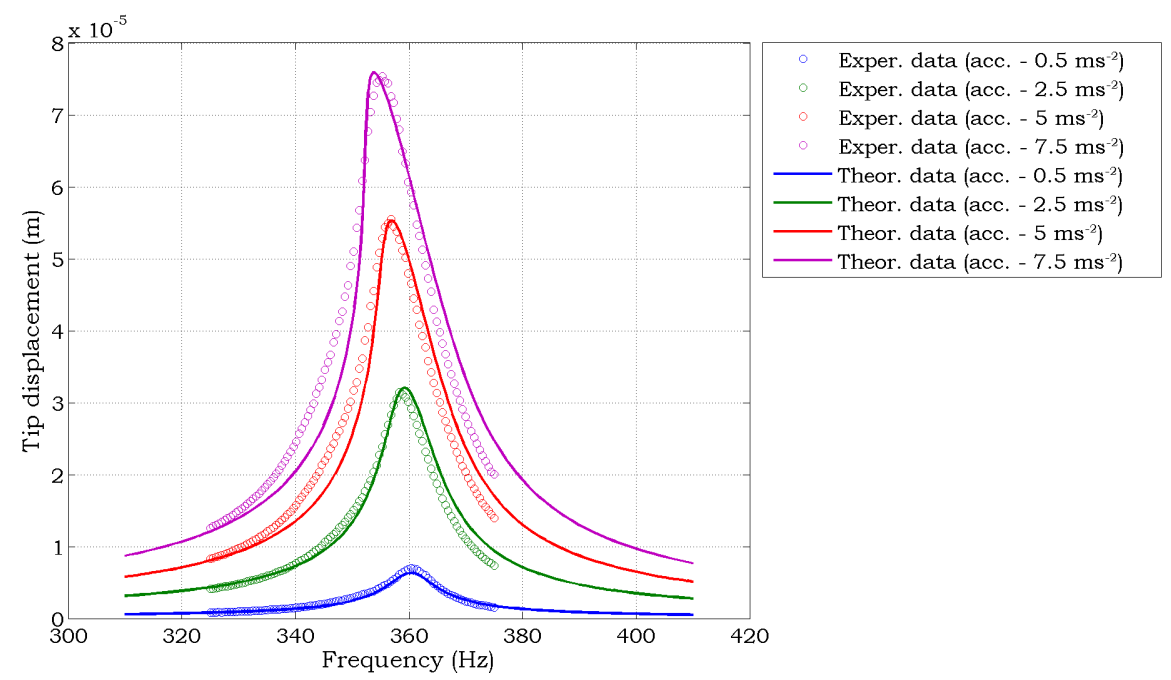

Figure 6: Comparison between theoretical and experimental tip displacement responses, for a conventionally designed energy harvester, in closed circuit conditions, subjected to various acceleration levels. Substrate non-linearity also included.

percentage difference between experimental and theoretical resonant frequencies, when subjecting the sample to a base acceleration of $7.5 \mathrm{~ms}^{-2}$, is $0.48 \%$. Note how, although peak magnitude is well predicted by the model, off-resonant behaviour is not. This is possibly due to imperfections in bonding during sample manufacture, with results from samples with shorter piezoelectric layers (Figures 8 and 9) showing better off-resonance matching. The variation in mechanical damping is again seen to resemble a quasi-linear increase with base acceleration, with data provided in Figure 7 for completeness.

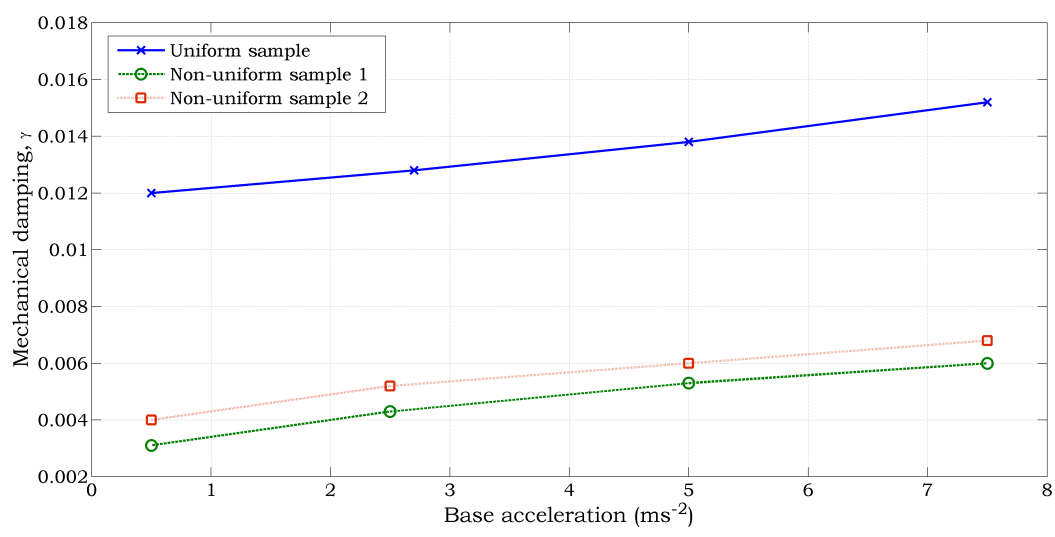

Figure 7: Dependency of mechanical damping on base acceleration for the three samples analysed in this section.

Results from samples comprising of shorter piezoelectric layers will now be shown in-order to demonstrate the versatility of the model. Figures 8 and 9 show results from the experimental testing and theoretical simulation of non-uniform samples 1 and 2 , 
respectively. It is important to note that no more curve fitting for non-linearity terms is undertaken here. The theoretical data in each case is obtained using the previously found $\mu_{1}$ and $\mu_{s 1}$ magnitudes of $-7 \times 10^{14} \mathrm{~Pa}$ and $-2 \times 10^{13} \mathrm{~Pa}$, respectively.

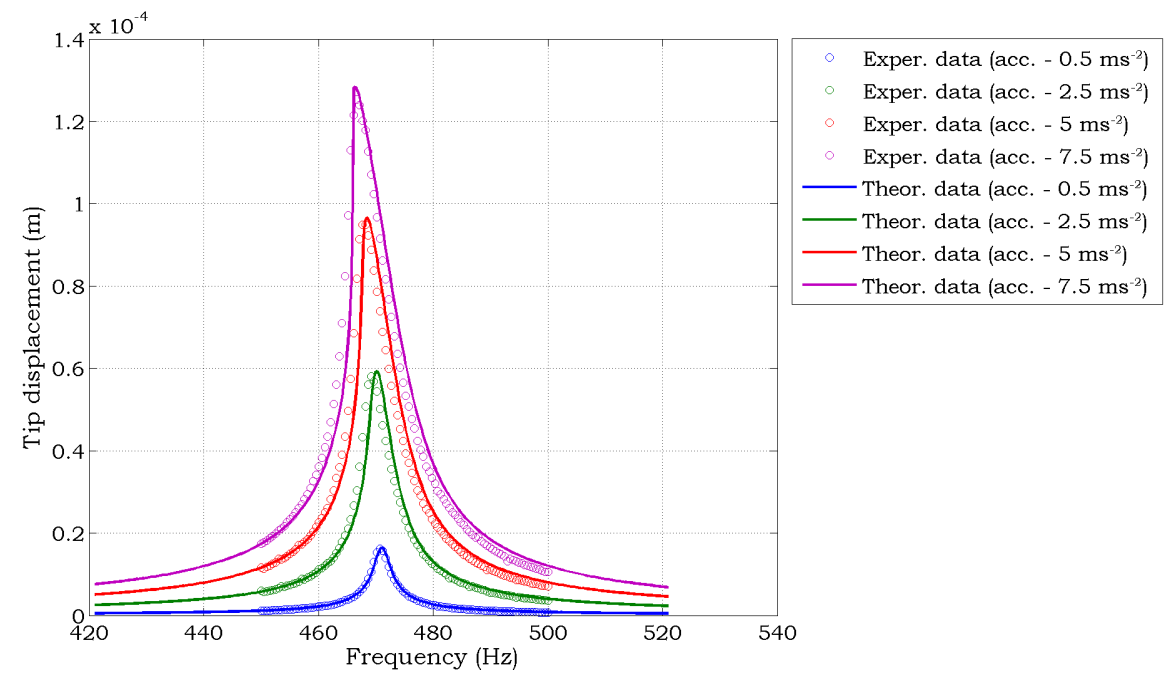

Figure 8: Comparison between theoretical and experimental tip displacement responses, for first non-uniform energy harvester, in closed circuit conditions, subjected to various acceleration levels. Substrate non-linearity also included.

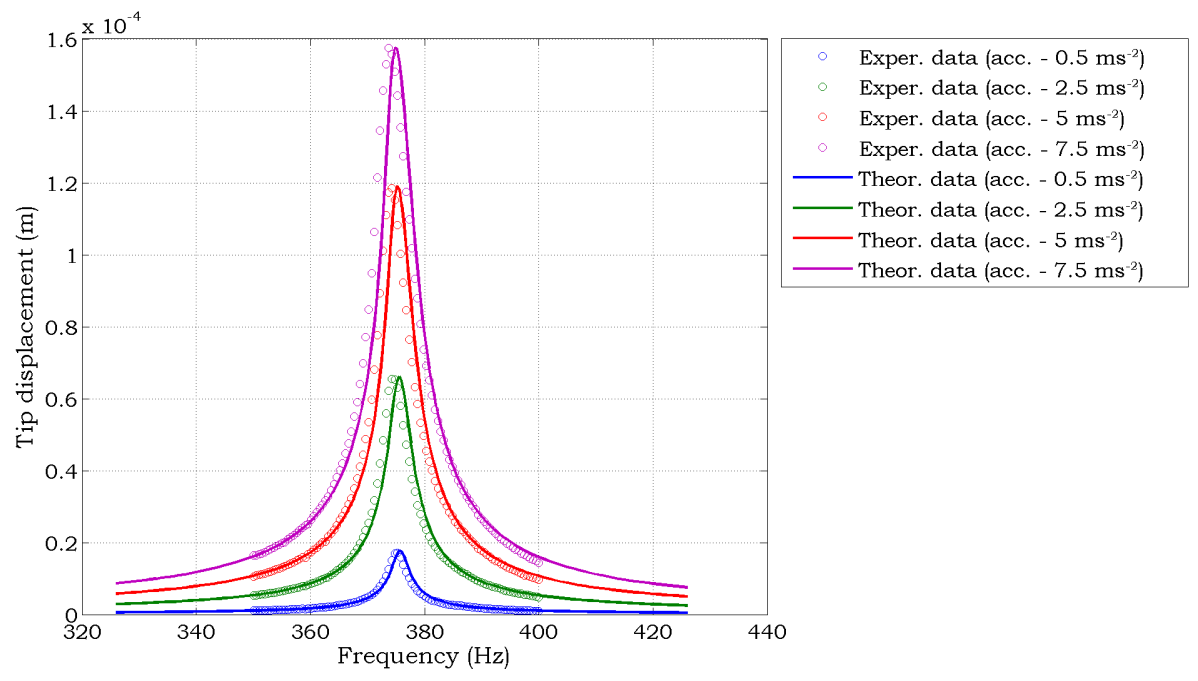

Figure 9: Comparison between theoretical and experimental tip displacement responses, for second non-uniform energy harvester, in closed circuit conditions, subjected to various acceleration levels. Substrate non-linearity also included.

Figures 8 and 9 show that good experimental-theoretical agreement is also obtainable for non-uniform samples using previously determined magnitudes for material non-linearity coefficients. The shift in resonant frequency between the two excitation 
extremes, in each case, is found to be $4 \mathrm{~Hz}$ and $1.6 \mathrm{~Hz}$, increasing with piezoelectric material length. Prediction of the resonant frequency by the theoretical model, at an acceleration of $7.5 \mathrm{~ms}^{-2}$, results in percentage errors of $0.06 \%$ and $0.29 \%$. This highlights the accuracy and versatility of the model present in Section 2 in predicting the behaviour of piezoelectric harvesters subjected to non-linear inducing excitation conditions. The mechanical damping was again found to increase in a quasi-linear manner with results presented in Figure 7. The occurrence of differing rates of increase in damping ratio with base acceleration $(26 \%, 70 \%$ and $94 \%$ for the uniform sample and non-uniform samples 1 and 2, respectively) emphasises the difficulties in predicting damping magnitudes. In the following section, the energy harvester will be connected to a load resistor in order to determine the magnitude of the remaining non-linear coefficient, $\mu_{2}$.

\subsection{Energy harvester connected to electrical load}

The magnitudes of $\mu_{1}$ and $\mu_{s 1}$ found from previous closed circuit testing are still valid as the sample tested here was manufactured from the same 'batch' of sheet piezoelectric material. The two variables which require estimation are $\mu_{2}$, found in Equation (2), and $\gamma_{r}(r=1)$, found in Equation (25). Dimensions for the sample under consideration are provided in Table 1 with a $45.02 \mathrm{~mm} \pm 1 \mathrm{~mm}$ extended length. The magnitude of mechanical damping $(0.0062,0.0085,0.0108$ and 0.0125$)$, corresponding to the results in Figure 10 at the 4 excitation levels, was obtained through closed circuit testing and curve fitting as previously demonstrated. Figure 10 provides results obtained when a $150 \mathrm{k} \Omega$ resistor is introduced to the system. The first observation to note is that good agreement between experimental and theoretical voltage responses is achievable without necessity of the non-linear coefficient $\mu_{2}$. Following this observation, the effects of $\mu_{2}$ on theoretical results are investigated, with realisation that $\mu_{2}$ has little influence on theoretical frequency responses. This is another difference when comparing the proposed theoretical model with that developed by Stanton et al. in [19], where the electro-elastic non-linear constant is utilised. It is believed that this coefficient can be assumed zero in the majority of energy harvesting scenarios due to the inherently low voltage levels. Applications utilising piezoelectric material for actuation are subjected to higher voltage levels and in these situations the non-linear coefficient, $\mu_{2}$, would have a more significant impact on theoretical results. As a final note, in terms of energy harvesting, the vision for the devices being proposed by Mutsuda et al. [21] is to generate power on the kilowatt scale and so analysis on these device would eventually require the predictions and utilisation of $\mu_{2}$.

From comparing the frequency shift for cases with and without a resistor, as one would expect, the level of experienced non-linearity reduces when energy is extracted from the system by the addition of an electrical load. The frequency shift (from an excitation of $0.5 \mathrm{~ms}^{-2}$ to $7.5 \mathrm{~ms}^{-2}$ ) reduces to $3.5 \mathrm{~Hz}$ from a $6.5 \mathrm{~Hz}$ shift obtained when the load resistor is excluded. Around $390 \mathrm{~Hz}$, there is noise in the experimental data in Figure 10. This is due to a resonant frequency from the clamp and shaker arrangement 


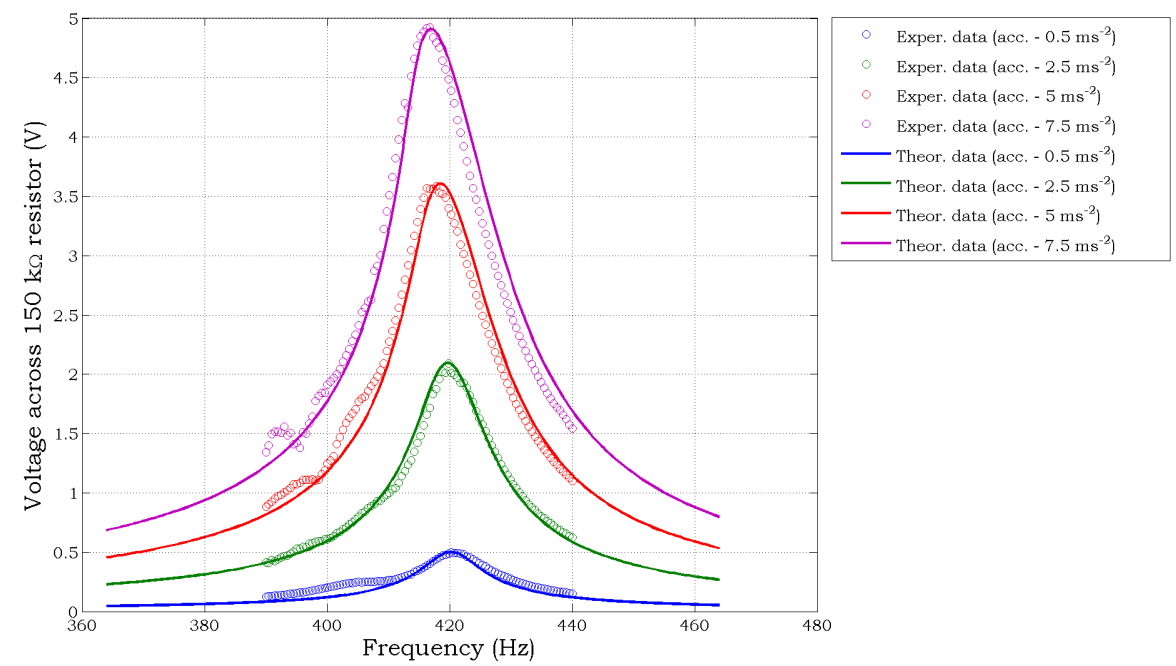

Figure 10: Comparison between theoretical and experimental voltage responses, for a conventionally designed energy harvester, connected to a $150 \mathrm{k} \Omega$ resistor, subjected to various acceleration levels. Substrate non-linearity also included.

interfering with vibrations of the energy harvesting device.

The non-linear constants obtained in this paper are only valid for this particular 'batch' of substrate and piezoelectric material. For each new 'batch' of material, experimental testing must be undertaken to determine the magnitudes of the three material non-linearity coefficients. It is advised that the following be undertaken to achieve this:

- A frequency sweep around the resonant frequency of the sample under investigation should be performed for at least 4 different base accelerations.

- It is advisable that testing of only substrate cantilevers be performed initially. This will eliminate all but one material non-linear coefficient, $\mu_{s 1}$, improving the fitting process. Tip displacement FRFs should be used here.

- Following this, it is suggested that a complete energy harvester sample (either uniform or non-uniform) be tested in closed circuit conditions. The fitting process should now be performed to obtain $\mu_{1}$, using the already determined $\mu_{s 1}$ magnitude. Again tip displacement FRFs should be used here. The fitting process can also be used here to obtain variations in mechanical damping with base acceleration.

- Finally, the testing of a complete energy harvester connected to load resistor can be undertaken. Voltage FRFs can be used in this case, with curve fitting used to provide the user with only remaining non-linear coefficient, $\mu_{2}$.

\section{Linear and non-linear model comparisons during device optimisation}

In this section, device optimisation is performed in relation to coverage of the piezoelectric layer. Theoretical results from both a linear and non-linear model will 
be provided and comparisons made. The width of both layers is taken to be $5 \mathrm{~mm}$ and the thickness of the substrate and piezoelectric layer taken to be $0.67 \mathrm{~mm}$ and $0.5 \mathrm{~mm}$ respectively. The material properties assigned to the device are given in Table 1 . The length of the substrate layer will remain fixed at $50 \mathrm{~mm}$ with the piezoelectric layer length varying from $50 \mathrm{~mm}$ to $1 \mathrm{~mm}$. Length reductions are made from the free end, i.e. $x_{1}$ remains 0 in Figure 1. Note how the Euler-Bernoulli assumptions made during the modelling procedure hold true as the overall beam length remains at $50 \mathrm{~mm}$ and only the length of the piezoelectric material is being reduced.

\subsection{Creating devices with identical fundamental frequency}

Before performing the study on piezoelectric coverage, a discussion on why a constant fundamental frequency approach is essential. Altering one geometric parameter alone is not advisable as this creates devices with differing fundamental frequencies. Say, the conventional device (both layers being the same length) is designed for an application to operate most effectively at $F \mathrm{~Hz}$, the dominant excitation frequency. Any changes to the piezoelectric layer length, whilst keeping all other geometric parameters constant, will alter the fundamental frequency creating an ineffective device. To avoid this mismatch between fundamental and dominant excitation frequencies across all designs, in this work the thickness of the substrate layer is used as a control parameter. Through this simple procedure, unbiased design comparisons can be made. Figure 11 shows the substrate thickness required for each piezoelectric layer length to create configurations with identical fundamental frequencies.

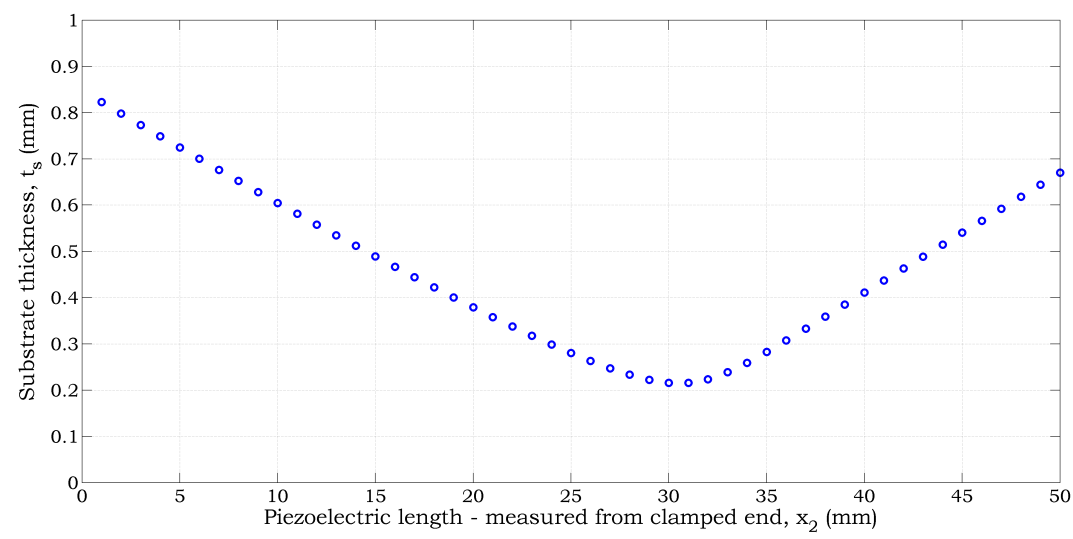

Figure 11: Plot indicating the substrate thickness required for designs with various piezoelectric layer lengths. All configurations have identical natural frequencies.

The data plotted in Figure 11 is obtained by sweeping through a range of substrate thickness for each design, plotting fundamental frequency vs. thickness, and interpolating to find an accurate value. The trend is as one would expect. As material is reduced from the free end, the mass of the device reduces at a greater rate than its stiffness. This causes an increase in fundamental frequency which must be 
countered by reducing substrate layer thickness. After a critical point, which depends on materials/initial size/inclusion of a tip mass, etc., the stiffness begins to reduce at a higher rate than device mass and so increases in substrate thickness are required.

\subsection{Piezoelectric length optimisation}

In this section the devices generated in Section 4.1 are subjected to an acceleration level of $7.5 \mathrm{~ms}^{-2}$ connected to a $1 \mathrm{M} \Omega$ resistor. The results obtained for the peak voltage, using both a linear and non-linear model, are shown in Figure 13-15. The linear results are obtained by setting the following time-independent constants, found in Equations (25) and (26), to zero $-C_{n 1}^{r} C_{n 4}^{r}, C_{n 6^{-}}^{r} C_{n 8}^{r}, C_{n 10}^{r}, C_{n 11}^{r}$ and $C_{n 13}^{r}$. For the non-linear model, non-linear constants, $\mu_{s} 1, \mu_{1}$ and $\mu_{2}$ obtained in Section 3.2 are used during the study.

The effect of variations in mechanical damping during optimisation studies is highly important and cannot be ignored. Detailed work on changes in mechanical damping, and predictions during piezoelectric coverage optimisation using a linear theoretical model, can be found in [27]. For the purpose of this work three scenarios will be considered:

- The damping magnitude will remain constant across all configurations.

- Damping data from both the uniform and 2nd non-uniform sample (PZT coverage of $100 \%$ and $21 \%$ respectively) is used to obtain a power relationship between device volume and damping ratio. Therefore in this scenario it is assumed that the magnitude of mechanical damping depends on device volume where reducing the volume by approximately $25 \%$ reduces the damping by $50 \%$. see the dash line in Figure 12 for numerical values.

- Experiments in [27] showed that predicting trends between mechanical damping and PZT length is extremely difficult. The author found that, overlying the general trend, large variations in damping occurred between samples; variations which were predominantly due to mounting and inconsistent clamping force. One observed trend between PZT length and mechanical damping showed a linear increase in damping with length until approximately $66 \%$ coverage, thereafter plateauing off for longer PZT lengths. By assuming the major contributor to damping magnitude to be the amount of adhesive, a similar trend can be used in this work by scaling according to data from the uniform sample in Figure 7. The final trend between damping ratio and length of PZT which will be used in the optimisation study is shown in Figure 12.

Figure 13 clearly shows differences in the performance trend obtained whilst using either a linear or non-linear model when a constant damping assumption is made. The linear model suggests that maximum voltage is generated when the piezoelectric material covers the entire beam, whereas the non-linear model suggests maximum voltage is generated for a device with $\mathrm{a} \approx 5 \mathrm{~mm}$ long piezoelectric layer. For cases where the piezoelectric layer is short the difference between model outputs is minimal and a 


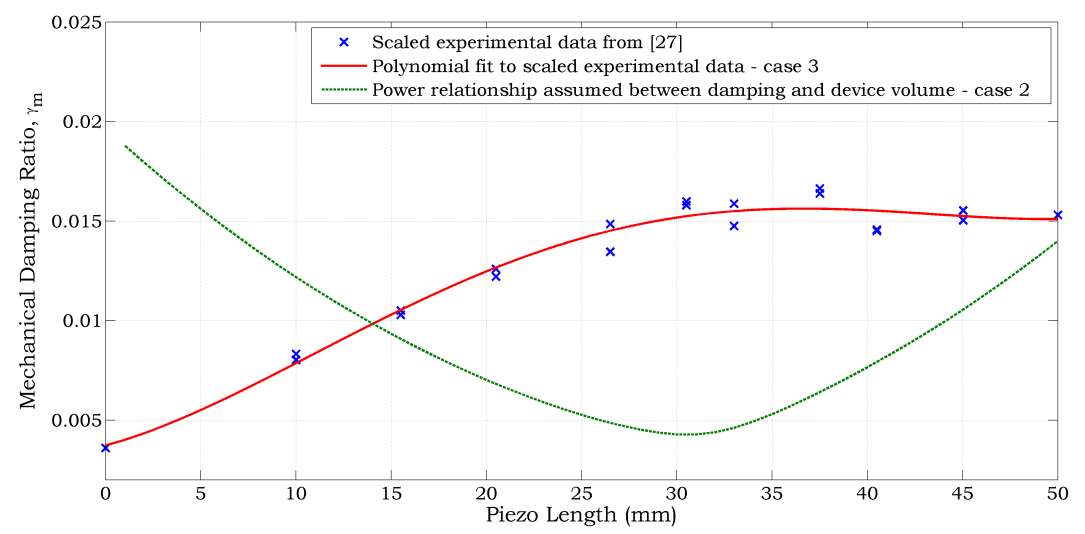

Figure 12: Two possible trends between mechanical damping ratio and piezoelectric material coverage (note substrate thickness is also changing in accordance with Figure 11). The first trend, represented by the dashed line, assumes a power relationship between damping and volume. The second, represented by the solid line, is obtained by scaling the findings found in [27].

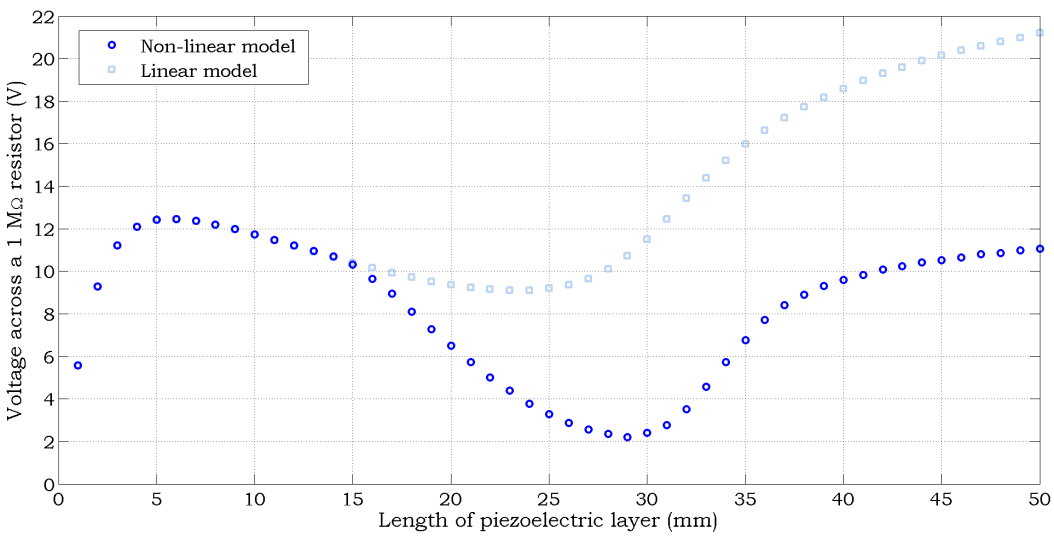

Figure 13: Trends between piezoelectric layer length and voltage across a $1 \mathrm{M} \Omega$ resistor for linear and non-linear models. Constant damping assumptions.

linear model would suffice. Due to the volume of piezoelectric material the effects from material non-linearity, which is the dominant effect, are reduced. However, as the material length increases, so too does the extent of non-linearity. This is the cause of observed discrepancies for devices with longer piezoelectric coverage. Note how for devices with the thinnest substrate layers, i.e. piezoelectric lengths of approximately $30 \mathrm{~mm}$ from Figure 11, large differences between model predictions exist. Naturally, the deflections experienced by these devices will be greatly increased which induces nonlinear behaviour thereby resulting in the relatively low voltage levels seen in Figure 13.

In Figure 14 results from both linear and non-linear models, while assuming damping varies through a power relationship with volume, are presented. The general trend is clearly very different from that obtained in Figure 13 where damping was assumed constant. Peak performance is seen to occur for devices with PZT coverage of 


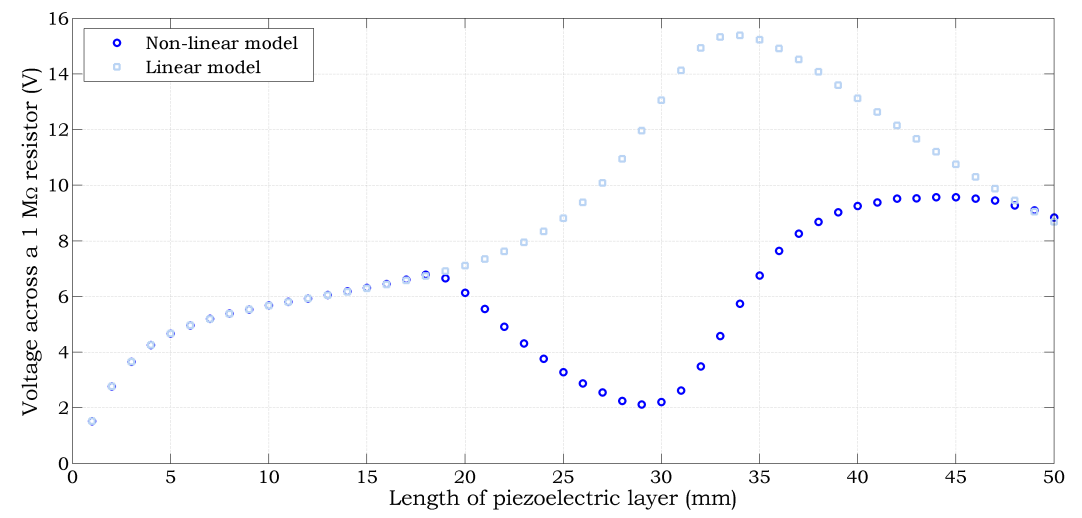

Figure 14: Trends between piezoelectric layer length and voltage across a $1 \mathrm{M} \Omega$ resistor for linear and non-linear models. Damping assumed to vary through power relationship with device volume, shown in Figure 12.

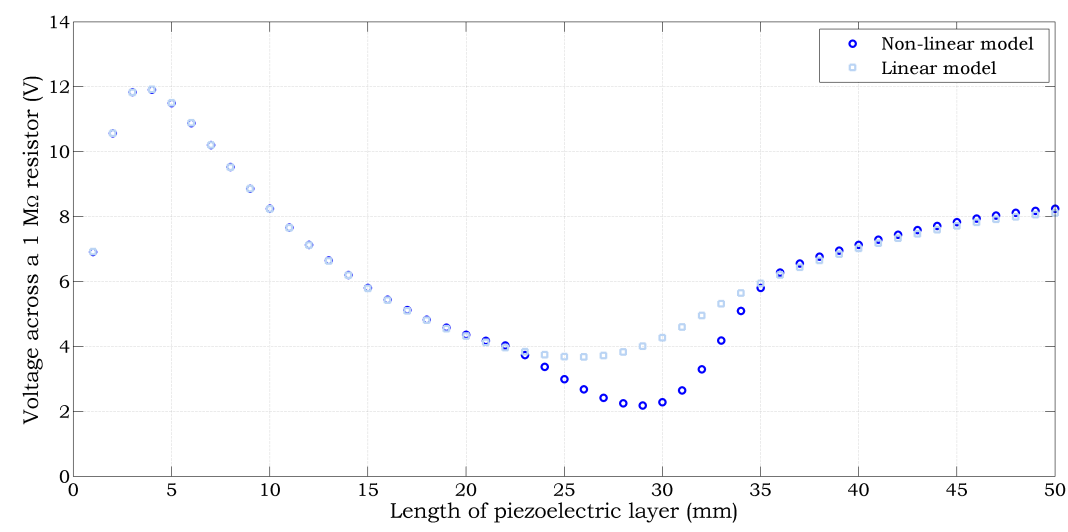

Figure 15: Trends between piezoelectric layer length and voltage across a $1 \mathrm{M} \Omega$ resistor for linear and non-linear models. Damping assumed to vary according to extrapolated data taken from [27], shown in Figure 12.

approximately $68 \%$ when utilising the linear model, and $100 \%$ coverage whilst utilising the non-linear model. For devices which exhibit low mechanical damping there is large divergence between the two model as a result of increased non-linearity in devices. In this case, devices with reduced PZT coverage are showing poor levels of performance due to the presence of large mechanical damping. Information on damping ratio magnitudes utilise here can be found in Figure 12.

When the damping magnitude is assumed to partly vary with adhesive length, in the manner shown in Figure 12, both models predict the same optimum configuration, see Figure 15. In this scenario, the samples with increased PZT coverage experience higher mechanical damping suppressing motion, thereby reducing the effects of non-linearity on peak performance. For this reason the differences between results from a linear model and a non-linear model are much smaller than those obtained when assuming a low constant damping ratio, Figure 13. The low mechanical damping for samples with 
reduced lengths of PZT is responsible for the observed spike in peak voltage. Similar trends, i.e. high performance from devices with short PZT layers, have previously been reported through experimental work in [27].

This brief study highlights the importance of using both a non-linear model with detailed knowledge on mechanical damping variations, during device optimisation in befitting excitation conditions. Although for the last tested case both models predicted the same optimum PZT coverage, the non-linear model is essential in predicting the resonant frequency at which peak performance occurs, see Figure 16. The device performance would have suffered by $5 \%$ if this shift was unknown to the energy harvester designer. In this example the shift, and detriment in performance, is small because the optimum configuration only has a $10 \%$ piezoelectric coverage, however, this will not always be the case.

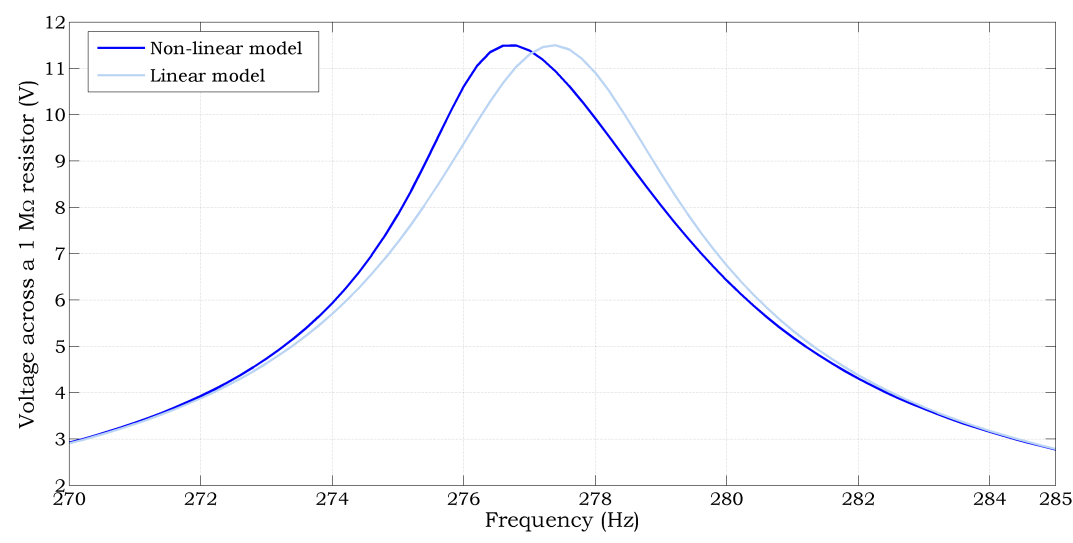

Figure 16: Frequency response functions obtained from linear and non-linear model for the optimum configuration in Figure 15.

\section{Conclusion}

A robust non-linear model to predict the dynamic response of piezoelectric cantilever energy harvesters has been developed and presented. Since piezoelectric material is known to behave in a non-linear fashion, even at moderate excitation levels, the inclusion of piezoelectric material non-linearity is seen as essential. Material non-linearity was also included for the substrate layer in addition to geometric non-linearity for the beam. The inclusion of substrate material non-linearity was important in terms of extending the applicability of the model and accounting for effects observed during experimental testing. Geometric non-linearity is realised by applying an in-extensibility condition, and material non-linearity is incorporated through the addition of higher order terms in constitutive equations. In order to ensure piezoelectric layer coverage can still be optimised, a transfer matrix model was developed allowing for accurate predictions in the eigenvalues and eigenvectors of segmented structures. Detailed model validation is provided through the use of harvesters manufactured in-house. This is then followed by 
comparing results from the non-linear model with a linear model while predicting the performance of energy harvesters.

Closed-circuit testing was initially undertaken which allowed for simplification of the fitting process when obtaining the magnitude of the non-linear material constants.

For this 'batch' of piezoelectric material a $\mu_{1}$ magnitude of $-7 \times 10^{14} \mathrm{~Pa}$ was found to provide adequate matching between experimental and theoretical data. This was seen to be the case across a range of samples each comprising of a different piezoelectric layer length. The devices were then attached to a load resistor in order to determine the magnitude of the second non-linear constant, $\mu_{2}$. It was concluded that the inclusion of this non-linear constant is not required when modelling harvester devices, possibly due to the low voltage levels associated with energy harvesting in comparison to when using piezoelectric material for actuation.

To determine the magnitude of the material non-linearity constant for the substrate layer, tests were performed in the absence of piezoelectric material. The magnitude of $\mu_{s 1}$ was found to be $-2 \times 10^{13} \mathrm{~Pa}$, which, as one would expect, is lower than that of the piezoelectric material. Another important finding was that mechanical damping increases in a quasi-linear manner with the base acceleration. This means that simple scaling, i.e. double the excitation to double the performance, does not strictly apply. Harvesters must be simulated in realistic operating conditions in order to accurately predict how they will perform.

A case study on device optimisation has also been presented here. Three different assumptions were made in regards to the mechanical damping variations with PZT length; (i) a constant damping ratio for all devices, (ii) a power relationship between damping and device volume, and (iii) a relationship obtained from a similar experiment undertaken in [27], extrapolated for this scenario. The results indicate that trends between piezoelectric layer coverage and performance are highly dependant on damping variations. The use of a non-linear model for estimating the optimum configuration in terms of peak power is debatable due to uncertainty in mechanical damping prediction. However, the model is essential for acquiring knowledge on the extent of changes in resonant frequency for the optimum configuration.

In terms of future work, experimental testing on harvesters with other material compositions is recommended. This will assist in gauging the applicability and limitations of the developed non-linear model. Testing on highly flexible devices such as those designed to be used in ocean applications, proposed by Mutsuda et al. [21], is currently in progress. Silicone rubber and PVDF materials make these devices more sensitive to geometric and substrate material non-linearities, which will reinforce the usefulness of the developed model.

\section{Acknowledgements}

This research was supported by the International Exchanges Scheme by the Royal Society of London, U.K.. 


\section{References}

[1] Mak K H 2011 Vibration Modelling and Analysis of Piezoelectric Energy Harvesters (University of Nottingham, UK) PhD Thesis

[2] Roundy S, Leland E S, Baker J, Carleton E, Reilly E, Lai E, Otis B, Rabaey J M, Wright P K and Sundararajan V 2005 IEEE Pervas. Comput. 4 28-36

[3] Elvin N and Erturk A 2012 Advances in Energy Harvesting Methods (New York, USA: Springer Science \& Business Media)

[4] Erturk A and Inman D J 2011 Piezoelectric Energy Harvesting (Chichester, UK: John Wiley \& Sons Ltd.)

[5] Sodano H A, Park G and Inman D J 2004 Strain 40 49-58

[6] Erturk A and Inman D J 2008 J. Vib. Acoust. 130041002 (15 pp)

[7] Erturk A and Inman D J 2009 Smart Mater. Struct. 18025009 (18 pp)

[8] Patel R, McWilliam S and Popov A A 2011 Smart Mater. Struct. 18025009 (18 pp)

[9] Tang L, Yang Y and Soh C K 2010 J. Intel. Mat. Syst. Str. 21 1867-189

[10] Ramlan R, Brennan M J and Mace B R 2008 ISMA Sep. 2008 (Leuven, Belgium)

[11] Daqaq M F 2010 J. Sound Vib. 329 3621-3631

[12] Ferrari M, Baù M, Guizzetti M and Ferrari V 2010 Sensor. Actuat. A-Phys. 172 287-292

[13] Tang L and Yang Y 2012 Appl. Phys. Lett. 101094102 (4 pp)

[14] Tang L, Yang Y and Soh C K 2012 J. Intel. Mat. Syst. Str. 23 1433-1449

[15] Masana R and Daqaq M F 2011 J. Vib. Acoust. 133011007 (10 pp)

[16] Joshi S P 1992 Smart Mat. Struct. 1 80-83

[17] Crespo da Silva M R M and Glynn C C 1978 J. Struct. Mech. 6 437-448

[18] Mak K H, Popov A A and McWilliam S 2012 J. Sound Vib. 331 2602-2623

[19] Stanton S C, Erturk A, Mann B P and Inman D J 2010 J. Appl. Phys. 108074903 (9pp)

[20] Stanton S C, Erturk A, Mann B P, Dowell E H and Inman D J 2012 J. Intel. Mat. Syst. Str. 23 183-199

[21] Mutsuda H, Watanabe R, Azuma S, Tanaka Y and Doi Y 2013 ASME 32nd ICOOAE June 2013 (Nantes, France)

[22] Pestel E C and Leckie F A 1963 Matrix Methods in Elasto-Mechanics (New York, USA: McGrawHill)

[23] Mahmoodi S N, Jalil N and Daqaq M F 2008 IEEE ASME T Mech. 13 58-65

[24] Dietl J M, Wickenheiser A M and Garcia E 2010 Smart Mater. Struct. 19055018 (12 pp)

[25] McCleary J 1995 Geometry from a Differentiable Viewpoint (Cambridge, UK: Cambridge University Press)

[26] http://www.mathworks.co.uk/ - Last accessed in July 2013

[27] Patel R 2012 Modelling, Analysis and Optimisation of Cantilever Piezoelectric Energy Harvesters (University of Nottingham, UK) PhD Thesis

[28] Malatker P 2003 Nonlinear Vibrations of Cantilever Beams and Plates (Virginia Polytechnic Institute, USA) $\mathrm{PhD}$ Thesis

[29] Yao L Q, Zhang J G, Lu L and Lai M O 2004 J. Microelectromech. S. 13 645-652

[30] Nouira H, Foltête E, Hirsinger L and Ballandras S 2007 J. Sound Vib. 305 243-260

[31] Rao S S 2004 Mechanical Vibrations - Fourth Edition (New Jersey, USA: Pearson Prentice Hall) 
Appendix A. Time-independent constants, $C_{n 1}$ through to $C_{n 14}$

$$
\begin{aligned}
& C_{n 1}^{r}=\left[\int_{0}^{L} W_{q}(s)\left[\frac{3}{2} K_{3}(s) W_{r}^{\prime \prime 2}(s)\right]^{\prime \prime} \mathrm{d} s\right] \\
& C_{n 2}^{r}=\left[\int_{0}^{L} W_{q}(s)\left[\left(K_{1}(s) W_{r}^{\prime \prime}(s) W_{r}^{\prime}(s)\right)^{\prime} W_{r}^{\prime}(s)\right]^{\prime} \mathrm{d} s\right] \\
& C_{n 3}^{r}=\left[\int_{0}^{L} W_{q}(s)\left[W_{r}^{\prime}(s) \int_{L}^{s} m(s) \int_{0}^{s}\left(W_{r}^{\prime \prime}(s) W_{r}^{\prime}(s)\right) \mathrm{d} s \mathrm{~d} s\right]^{\prime} \mathrm{d} s\right] \\
& C_{n 4}^{r}=\left[\int_{0}^{L} W_{q}(s)\left[W_{r}^{\prime}(s) \int_{L}^{s} m(s) \int_{0}^{s}\left(W_{r}^{\prime 2}(s)\right) \mathrm{d} s \mathrm{~d} s\right]^{\prime} \mathrm{d} s\right] \\
& C_{n 5}^{r}=\left[\int_{0}^{L} W_{q}(s)\left[\frac{1}{2} K_{4}(s)\right]^{\prime \prime} \mathrm{d} s\right] V(t) \\
& C_{n 6}^{r}=\left[\int_{0}^{L} W_{q}(s)\left[\left(K_{2}(s)\right) W_{r}^{\prime \prime}(s)\right]^{\prime \prime} \mathrm{d} s\right] \\
& C_{n 7}^{r}=\left[\int_{0}^{L} W_{q}(s)\left[\frac{1}{2} K_{4}(s) W_{r}^{\prime \prime}(s) W_{r}^{\prime}(s)\right]^{\prime} \mathrm{d} s\right] \\
& -\left[\int_{0}^{L} W_{q}(s)\left[\frac{1}{4} K_{4}(s) W_{r}^{\prime 2}(s)\right]^{\prime \prime} \mathrm{d} s\right] \\
& C_{n 8}^{r}=\left[\int_{0}^{L} W_{q}(s)\left[\left(\left(K_{2}(s)\right) W_{r}^{\prime \prime}(s) W_{r}^{\prime}(s)\right)^{\prime} W_{r}^{\prime}(s)\right]^{\prime} \mathrm{d} s\right] \\
& C_{n 9}^{r}=\left[\iiint_{V_{s}} \rho_{s} W_{q}(s) \mathrm{d} V+\iiint_{V_{p}} \rho_{p} W_{q}(s) \mathrm{d} V\right] \\
& C_{n 10}^{r}=\left[\int_{0}^{L} K_{2}(s) W_{r}^{\prime \prime 2}(s) \mathrm{d} s\right] \\
& C_{n 11}^{r}=\left[\int_{0}^{L} 2 K_{2}(s)\left(W_{r}^{\prime \prime 2}(s) W_{r}^{\prime 2}(s)\right) \mathrm{d} s\right] \\
& C_{n 12}^{r}=\left[\int_{0}^{L} \frac{1}{2} K_{4}(s) W_{r}^{\prime \prime}(s) \mathrm{d} s\right] \\
& C_{n 13}^{r}=\left[\int_{0}^{L} \frac{3}{2} K_{4}(s)\left(\frac{1}{2} W_{r}^{\prime \prime}(s) W_{r}^{\prime 2}(s)\right) \mathrm{d} s\right] \\
& C_{n 14}^{r}=\left[\int_{0}^{L} K_{5}(s) \mathrm{d} s\right]
\end{aligned}
$$

